\title{
Competencias ciudadanas con juegos digitales para niños y niñas
}

Enviado: 4 de febrero de 2020 / Aceptado: 15 de junio de 2021 / Publicado: 31 de diciembre de 2021

ANA DOLORES VARGAS SÁNCHEZ

Facultad de Educación, Universidad de La Sabana, Colombia ana.vargas@unisabana.edu.co

(D) 0000-0002-5633-0901

JENNY ISABEL BARAJAS AVILA

Colegio Cedid San Pablo de Bogotá, Colombia jennyisabarajas@hotmail.com

DOI 10.24310/IJNE.8.2021.11858

\section{RESUMEN}

Este artículo presenta una investigación que buscó determinar las contribuciones de una estrategia pedagógica apoyada en juegos digitales, para el fortalecimiento de estándares de competencias ciudadanas, en niños de tercer grado de Bogotá, Colombia. El estudio se desarrolló bajo un enfoque cualitativo y con un diseño de estudio de caso. El análisis de resultados indicó que los juegos digitales en la formación de competencias ciudadanas hacen que los alumnos adquieran conocimientos e información para interactuar de manera asertiva en su grupo social y en el aula. No obstante, los juegos digitales no garantizan que los alumnos transfieran sus conocimientos al contexto real en el que viven.

Palabras Clave: juegos educativos, educación primaria, competencias ciudadanas.

\section{ABSTRACT}

Citizenship competitions with digital games for children

This article presents an investigation that sought to determine the contributions of a pedagogical strategy supported by digital games for the strengthening of citizen competencies standards, in third grade children from Bogotá, Colombia. This research was developed under a qualitative approach and with a case study design. The analysis of results indicated that digital games in the formation of citizen competences. This makes students acquire knowledge and information to interact assertively in their social group and in the classroom. However, digital games do not guarantee that students transfer their knowledge to the real context.

Keywords: educational games, primary education, citizen competencies. 


\section{INTRODUCCIÓN}

La formación para la ciudadanía es un factor fundamental en los procesos educativos, teniendo en cuenta que hace parte de la capacitación integral que todo estudiante debe recibir, aún más en la edad escolar, en la cual los niños fortalecen todas sus dimensiones del desarrollo (Muñoz, 2018; Urrea Monclús, et al., 2018; Quiceno, Betancur \& Rojas, 2020). Es así como, el Ministerio de Educación Nacional (MEN) de Colombia, plantea la importancia de "formar ciudadanos con valores éticos, respetuosos de lo público, que ejerzan los derechos humanos, cumplan sus deberes sociales y convivan en paz" (Ministerio de Educación Nacional, 2012, p. 1), siendo también una prioridad a nivel mundial y considerándose un desafío para toda la sociedad.

Los espacios de formación para la ciudadanía abarcan todos los contextos en los cuales se desarrollan los niños y niñas, entre estos el hogar, los medios de comunicación, la calle e instituciones educativas, y más ahora, el Internet, donde aprenden a vivir en comunidad, a trabajar en grupo, a identificarse, reconocer características, diferencias e interactuar con sus pares es fundamental (MEN, 2004).

Adicional, dentro del contexto bogotano, los índices de agresividad escolar se han mantenido en niveles elevados, tal como lo ha reportado la Secretaría de Educación Distrital, en el 2016 se han alcanzado 30.241 casos que alteraron el clima escolar y vulneraron los derechos en las instituciones, siendo Bosa (4.078) la localidad con mayores casos reportados (Universidad Distrital Francisco José de Caldas, 2016). Existiendo esta necesidad no sólo en Colombia, sino a nivel internacional, donde la UNESCO (2018) reporta, por ejemplo, que en 63 países los estudiantes han experimentado algún tipo de castigo corporal, así como menciona que las niñas y los niños tienen las mismas probabilidades de sufrir acoso. Pero, en Oriente Medio, África del Norte y el Pacífico, los niños son más propensos que las niñas, a ser intimidados. La apariencia física es considerada el aspecto que los estudiantes indican como más constante, al ser acosados, un 15,3\% reporta que ha recibido burlas sobre el aspecto de su cara o su cuerpo.

En este contexto, se estructuró un espacio de investigación con el objetivo de identificar las contribuciones de una estrategia pedagógica, apoyada en juegos digitales, para el fortalecimiento de estándares de competencias ciudadanas. Este proceso nace en el marco del proyecto profesoral "Las TIC en la educación de la paz y la convivencia" de la Maestría en Proyectos Educativos mediados por TIC del Centro de Tecnologías para la Academia de la Universidad de La Sabana, Colombia. 


\subsection{Competencias Ciudadanas}

Para Colombia, las competencias ciudadanas son claves en el sistema educativo, como lo ha propuesto el Ministerio de Educación Nacional al formular políticas, planes y programas enfocados a la formación de ciudadanos, y quienes las definen como "el conjunto de conocimientos y de habilidades cognitivas, emocionales y comunicativas que, articulados entre sí, hacen posible que el ciudadano actúe de manera constructiva en la sociedad democrática” (MEN, 2012, p.1).

Las competencias ciudadanas se orientan a través de unos estándares básicos en los cuales se plantea que es importante "considerar la formación ciudadana como un proceso que se puede diseñar, con base en principios claros, implementar, con persistencia y rigor, evaluar continuamente e involucrar en los planes de mejoramiento de cada institución" (MEN, 2004, p. 5). Estos estándares se organizan en tres grupos: convivencia y paz; participación y responsabilidad democrática; pluralidad, identidad y valoración de las diferencias (MEN, 2004).

Para la presente investigación se tomó en cuenta el grupo convivencia y paz, el cual se fundamenta en la consideración de los demás y de cada persona como ser humano. De forma específica se apoyaron dentro de la investigación los indicadores de:

- "Identifico como me siento yo o las personas cercanas cuando no recibimos buen trato y expreso empatía, es decir, sentimientos parecidos o compatibles con los de otros" (MEN, 2004, p. 16).

- "Comprendo que las normas ayudan a promover el buen trato y evitar el maltrato en el juego y en la vida escolar" (MEN, 2004, p. 16).

Investigadores como Ruiz y Chaux (2005) presentan el grupo de convivencia y paz, centrado en la formación ciudadanía donde "los ciudadanos sean capaces de convivir con los demás de manera pacífica y constructiva" (p. 57-58), lo cual no significa ausencia de conflictos, sino que se manejan sin agresión y buscando favorecer los intereses de las partes involucradas.

A partir de lo anterior, para esta investigación se considera el buen trato como la forma en que se relacionan las personas, basándose en sentimientos de respeto y valoración hacia la dignidad del otro, caracterizado por hacer uso de la empatía, es decir, de la comprensión de los sentimientos, emociones y necesidades propias y de los demás; así como de la comunicación entre las personas, lo cual deja claro que el buen trato se desarrolla y se aprende (Reyes, 2015).

Además, debe considerarse como un proceso que inicia desde la infancia y depende en gran medida de los diferentes entornos en los cuales crece cada persona, y donde se deben 
definir normas o límites claros, para llegar al logro de una convivencia pacífica (Reyes, 2015; Roca, 2010; Montes y Montoya, 2003, citado en Muñoz y Mondaca, 2007).

\subsection{El Aprendizaje Basado en Juegos: una opción que lleva a la comprensión}

Considerar nuevas estrategias para fortalecer las competencias ciudadanas, lleva a contemplar las Tecnologías de la Información y de la Comunicación (TIC) como una estrategia innovadora en los procesos de enseñanza y aprendizaje, ante lo cual la UNESCO (2013) plantea en el marco de los avances tecnológicos que:

Aprender a vivir juntos: las TICs, como medio de comunicación, nuevamente el uso ético de las TICs, las redes sociales, el trabajo cooperativo, las producciones colectivas, espacios de participación social, desarrollo de ciudadanía, entre otros, todo lo cual aporta a la cultura de la paz. En este sentido, el uso de TICs en educación no implicaría sólo promover el intercambio e interacción, sino que debe contribuir a visibilizar y valorar la diversidad cultural desde un enfoque de derechos humanos (p. 26).

De ahí, que las herramientas tecnológicas proporcionan a los docentes métodos alternativos para motivar a los estudiantes y mejorar los procesos de aprendizaje con el uso de multimedia, hipertextos, sistemas interactivos, así como recursos didácticos, de acuerdo con las necesidades de los estudiantes en las diferentes áreas del conocimiento (Pizarro y Cordero, 2013; Roig, et al., 2015; Rosi, et al., 2016).

De acuerdo a lo anterior, se encuentran los juegos digitales como parte de las herramientas tecnológicas referidas para la motivación (gamificación) y mejora de los proceso de enseñanza y aprendizaje, de manera que el Aprendizaje basado en Juegos o Game Based Learning (GBL), reside en el uso pedagógico y reflexivo de los juegos y videojuegos, los cuales han sido diseñados no solo con fines de entretenimiento, sino de aprendizaje, acercándose a situaciones reales (Santiago, et al., 2015; Cha, et al., 2008; Gros, 2008; Rodríguez y Santiago, 2015; Ojeda, et al., 2020).

De esta forma, este estudio tuvo como finalidad identificar las contribuciones de una estrategia pedagógica apoyada en juegos digitales, para el fortalecimiento de dos estándares de competencias ciudadanas del grupo de convivencia y paz, concernientes al sentimiento de empatía y manejo de normas para el buen trato, en estudiantes de tercer grado en un colegio público de Bogotá.

Para ello se buscó inicialmente detectar las características de los estudiantes, en relación con el manejo de normas para el buen trato y el sentimiento de empatía en la vida escolar, 
para luego diseñar e implementar la estrategia. Una vez que se implementó, se analizó cómo fue la experiencia de los estudiantes con la estrategia pedagógica apoyada por juegos digitales, para así dar pautas a la institución sobre el desarrollo de un proyecto educativo para el fortalecimiento de competencias ciudadanas apoyadas en las TIC.

\section{METODOLOGÍA}

Esta investigación es de enfoque cualitativo, tomando en cuenta que el conocimiento y la comprensión de la realidad se basa desde el diario vivir (Hernández, et al., 2010; Gómez, 2006). Varios autores (Punch, 2014; Lichtma, 2013; Morse, 2012, citados por Hernández, et al., 2010) mencionan que este tipo de estudios sólo se realiza cuando el objetivo está centrado en explorar la experiencia y percepción de los participantes de un fenómeno, buscando profundizar en las interpretaciones y significados que se generan.

El diseño de la investigación se orientó al estudio de casos, debido a que se tenía interés en profundizar sobre cómo funcionaba cada una de las partes de la estrategia pedagógica y las interacciones con los estudiantes (Stake, 1998). Buscando comprender, escuchar, observar e interpretar, más allá que generalizar, tomando en cuenta que el caso no estaba aislado, sino que hacía parte de un contexto propio que le afectaba e influía sobre las acciones que se realizaban (Stake, 1988). Por lo que cabe aclarar que la intención del estudio se concentró en establecer relaciones entre la estrategia pedagógica y la adquisición de conocimientos sobre las competencias ciudadanas (buen trato y empatía). Este estudio se llevó a cabo desde el 2015 hasta el 2017.

Entre las limitaciones de la presente investigación, se evidenció la importancia de profundizar más en torno a la categoría emergente del Internet como factor distractor, lo cual ha sido referenciado por otras investigaciones (Wood, et al., 2008; Wood, et al., 2012) que también lo han llamado desarrollo de una actividad de multitarea que los niños y niñas realizan.

\subsection{Participantes}

La población participante estuvo conformada por los estudiantes de la jornada de la tarde de un colegio público de Bogotá, ubicado en la localidad de Bosa. Con un grupo constituido por 18 niños y 24 niñas con edades entre los 8 y 10 años, pertenecientes al tercer grado de estratos socioeconómicos bajo (2) y bajo-bajo (1), lo que significa que la población ha sido clasificada por el Departamento Administrativo Nacional de Estadística (DANE), con escasos recursos y habitando en vividas considerados tugurios o en alquiler (DANE, 2017). 
Tomando como criterios de selección el grupo de estudiantes que presentó mayor número de problemas convivenciales, bajo desempeño en áreas afines a las competencias ciudadanas y falta de formación sobre las mismas.

\subsection{Técnicas e instrumentos}

Como técnicas de recolección de datos se utilizaron algunas fuentes de información primaria, entre estas la observación, la entrevista y la encuesta, para conocer las particularidades del problema en estudio (Gómez, 2006, p. 60). Todos los instrumentos de investigación diseñados se validaron en un proceso de pilotaje con estudiantes y a través de la validación de expertos.

\subsection{Estrategia pedagógica: los valores básicos de la convivencia ciudadana}

Los valores básicos de la convivencia ciudadana es el nombre dado a la estrategia pedagógica, la cual estuvo conformada por diez actividades distribuidas en ocho sesiones y dos preliminares (ver Figura 1), con duración de una hora y quince minutos por semana. En cada una de las actividades se contó con tres momentos de ejecución: 1. interacción con el juego digital, 2. trabajo grupal e individual con sopa de letras, crucigramas y test online, y 3. reflexión a modo de diálogo que no incluyó el uso de TIC (mesas redondas, representaciones, invención de cuentos).

Figura 1. Secuencia de actividades de la estrategia pedagógi ca. Fuente: Elaboración propia

Sensibilización
(Sesión Preliminar) Actividades previas

10 - International Journal of New Education | Núm. 8 
Todas las actividades fueron orientadas desde el sitio web principal del proyecto http://jennyisabarajas19.wix.com/aprendiendoyjugando

Las primeras dos actividades buscaron sensibilizar y acercar a los estudiantes a la finalidad de la estrategia pedagógica, las actividades tres y cuatro se enfocaron en fortalecer lo correspondiente con el estándar "identifico cómo me siento yo o las personas cercanas cuando no recibimos buen trato y expreso empatía” (MEN, 2004, p. 16), mientras que las actividades de la cinco a la diez se concentraron en el estándar "comprendo que las normas ayudan a promover el buen trato y evitar el maltrato en el juego y en la vida escolar" (MEN, 2004, p. 16).

Es importante resaltar que los dos estándares de competencias ciudadanas trabajados estuvieron relacionados en el marco de la formación para la convivencia y la cultura de paz, lo que permitió que las actividades de cada una de las sesiones fortalecieran ambos estándares, a pesar de estar clasificados por temática.

\section{RESULTADOS}

A continuación, se presentan los resultados obtenidos desde las categorías a priori planteadas: normas de convivencia, trato hacia los demás, habilidad de empatía y rol de los juegos digitales.

\subsection{En relación con las normas de convivencia aplicadas en el aula de clases}

Previo a la implementación se identificó que se contaban con normas de convivencia establecidas por el profesor, en las cuales los estudiantes no participaron. Además de evidenciarse la poca aplicación de las normas en la vida escolar por parte de los estudiantes, ya que esperaban de manera constante las indicaciones por parte del profesor, aspecto que estudiantes y el director de grupo refirmaron, haciendo mención a "no siempre se evidencia la aplicación de las normas, se les tiene que recordar en todo momento" (extracto de entrevista inicial al director de curso).

Una vez que se implementa la estrategia con juego digitales, se abarcaron normas relacionadas con la convivencia en el entorno escolar (no interrumpir al otro, compartir, pedir permiso), así como aquellas que involucraban el fortalecimiento de habilidades sociales básicas (pedir la palabra, escuchar, dar las gracias, respetar el turno, disculparse). Buscando que se caracterizaran por ser realizables, de carácter preventivo y medibles, lo que son aspectos destacados por distintos expertos (Torrego y Moreno, 2003; Chaux, Vargas, Ibarra y Minski, 2013 citado por Ministerio de Educación Nacional de Colombia, 2014; Jares, 2001). 
Lo que llevó a que se observara en los estudiantes la adquisición de conocimientos sobre las normas, pero la práctica de estas es un aspecto que requiere de continuidad para que sea interiorizado en la vida escolar, lo anterior debido a que algunos de los estudiantes aún les constaba aplicarlas, o simplemente recordarlas.

A través de los registros de observación se pudo evidenciar que normas como no interrumpir al otro, compartir y pedir permiso, fueron las que comenzaron a aplicar a medida que la estrategia avanzaba, lo cual también se hizo evidente en los espacios de reflexión donde, por ejemplo, analizando uno de los juegos digitales utilizados, dice la niña estudiante 7 "si yo peleo con alguien, me disculpo" (extracto de observación).

\subsection{En relación con el trato hacia los demás}

Se halló que las manifestaciones de buen trato o respeto entre los estudiantes eran escasas en un día escolar, primaban las burlas entre compañeros y los juegos entre los niños y niñas eran bruscos, llegando a maltratarse y agredirse, en una de las observaciones iniciales se pudo identificar que "el estudiante 4 le da un puño al estudiante 2, se les llama la atención, dicen que estaban jugando" (extracto observación sesión preliminar). Lo que se reafirma en las entrevistas, donde una estudiante indica al preguntarle sobre ¿cuál era su apreciación con relación a las faltas cometidas en su salón de clases? "se pegan muy duro, son bruscos, se agreden” (extracto entrevista estudiante).

Al aplicar la estrategia, algunos de los juegos buscaban que los estudiantes analizaran y reflexionaran sobre situaciones cercanas a su contexto, esto permitió documentar la apropiación de la temática abordada al tener que darles solución a las situaciones presentadas, tal como lo mencionó uno de los niños "el juego permitió saber sobre el respeto y el juego de roles para ponerse en el lugar de los demás” (extracto observación 2).

En una de las sesiones de reflexión, al preguntarles a los niños sobre qué decisión tomaron sobre la situación presentada en el juego, ellos expresaron:

La profesora les pregunta ¿qué decisión tomaron ustedes?, la estudiante 1 dice "que no pelearan”, el niño 8 dice "que sigan siendo amigos", la niña 7 dice "que no se digan groserías entre ellos", el estudiante 4 dice "uno no pelea, sino que se disculpa", la estudiante 9 dice "cuando uno tiene amigos nunca tiene que pelear" (extracto observación 3).

Pero aspectos relacionados con aceptar las ideas y opiniones de los demás, necesitan de mayor acompañamiento y formación, así como lo relacionado con el trato entre los estudiantes durante los periodos de juego (descanso), buscan prevenir que la agresión no se vea como algo natural dentro de los espacios de ocio. 
Lo anterior se identificó, debido a que en las observaciones y las entrevistas se evidenciaron aspectos contradictorios, por ejemplo, para algunos niños ya no se presentaban juegos bruscos, el estudiante 6 manifestó "ya nunca lo hacemos" (extracto entrevista final estudiantes), a pesar de que dentro del aula se observaron juegos agresivos hasta la última sesión.

\subsection{Con relación a la habilidad de empatía en los estudiantes}

La habilidad de empatía involucra un proceso de comprensión de los sentimientos y emociones, la preocupación por los demás y la ayuda hacia los demás, desde esta mirada se analizó la estrategia con juegos digitales.

$\mathrm{Al}$ iniciar el proceso de investigación se pudo evidenciar que algunos de los estudiantes se preocupaban por sus compañeros en situaciones que involucraban un mal trato, lo cual comunicaban a la profesora titular del curso. Pero cuando alguno de los compañeros necesitaba ayuda, no fueron frecuentes las expresiones de colaboración.

En la entrevista con la profesora titular ella mencionó "si, por lo general cuando el sentimiento es de tristeza, ellos se solidarizan y muestran cariño" (extracto entrevista inicial director de curso), mientras que una de las estudiantes expresa "a veces les importa, a veces no" (extracto entrevista inicial estudiante), reafirmando el hecho de que no todos brindan manifestación de comprensión hacia el otro.

Y al preguntarles a un grupo de diez niños ¿Qué hacían si observan que tratan mal a alguno de tus compañeros? El $40 \%$ (4) indico que le agrada y se une al resto del grupo, pero se resalta que el $60 \%(6)$ de los encuestados indico que le molestaría y buscaría detener la situación, o buscaría calmar a su compañero (ver Gráfico 1).

Gráfico 1. ¿Qué haces si observas que tratan mal a alguno de tus compañeros? Fuente: Elaboración propia

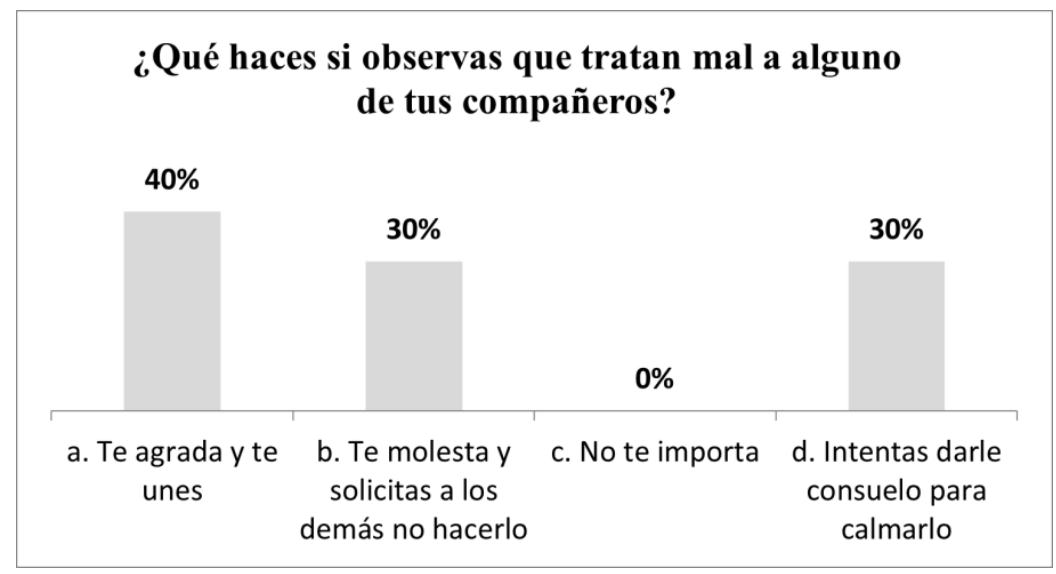


Durante la implementación de la experiencia se encontró que fue fortalecida la empatía desde las situaciones presentadas en cada una de las herramientas tecnológicas, y a nivel vivencial a partir de la realización de actividades en grupo y en los instantes de reflexión. Con relación a la comprensión de los sentimientos y a las emociones (alegría, tristeza, miedo, rabia) fortalecidas dentro de la estrategia pedagógica, se identificó que los estudiantes lograron identificarlas en sí mismos y en los demás.

Luego de observar un video, la profesora pregunta sobre la idea o enseñanza principal, la estudiante 20 dice "ayudar a los demás", el estudiante 15 dice "ayudar a las personas cuando se caen”, la estudiante 24 dice "ayudar y no ser grosero con las personas y ayudar a quien necesite ayuda" (extracto observación 6).

En lo referido a la ayuda hacia los demás, fue una de las variables que mayores resultados positivos presentó, teniendo en cuenta que los estudiantes aplicaron los conceptos aprendidos en cada uno de los momentos de la experiencia, aumentando las acciones de ayuda hacia sus compañeros con el uso de recursos digitales y en los espacios de trabajo en grupo y de reflexión, lo cual es coherente con lo que afirma "los niños se irán formando en la madurez emocional a medida que los adultos les enseñen y practiquen con ellos. Dichas habilidades se pueden aprender aprovechando cada momento cotidiano en el hogar o en el aula" (Asociación Española Contra el Cáncer AECC, 2010., p. 8).

Dichas expresiones de preocupación por los demás se observaron en varias de las sesiones, tales como:

"el niño estudiante 4 esta triste desde el momento en que vieron la animación y el niño estudiante 8 lo abraza y hablan (...) el niño estudiante 4 le dice a la profesora que el equipo de la niña estudiante 5 no le suena” (extracto observación 3).

Asimismo, se les solicita a los estudiantes en la encuesta final indicar un compromiso personal, tomando en cuenta todo lo aprendido a través de la estrategia, algunos de ellos indican:

"La estudiante 1 expresa "voy a respetar a las personas", el estudiante 12 "voy a respetar", el estudiante 4 "voy a cuidar el ambiente", la estudiante 17 "voy a ayudar a las personas" (extracto cuestionario).

\subsection{En relación con el rol de los juegos digitales en las Competencias Ciudadanas}

La categoría de rol de los juegos digitales fue abordada desde el análisis de las variables de actitud y acceso. Pero también tomando en cuenta que no solo eran medios de entretenimiento, sino medios con fines pedagógicos y de aprendizaje (Santiago, Trabaldo, Camijo y Fernández, 2015). 
En lo referido a la actitud de los niños y niñas hacia los juegos digitales, se encontraron expresiones de agrado, gusto, interés y participación, seguían las indicaciones, concentrados y motivados por explorar nuevos retos, aunque sólo uno de los niños en una de las sesiones indicó "que aburrido otra vez juegos" (extracto registro de observación sesión 2), pero al avanzar las sesiones su percepción cambió y jugó con interés. Algunos de los niños y niñas mencionan:

Estudiante 2 dice "las imágenes son muy chistosas", las estudiantes 13, 6 y 30 juegan concentradas, el niño estudiante 24 dice "que divertido", el estudiante 20 va avanzando y dice "es divertido" (extracto observación 2).

Tomando en cuenta la percepción de los estudiantes hacia los juegos digitales se puede establecer que estos pueden motivar y acercar a temáticas que encierran las competencias ciudadanas.

Con relación al acceso a los juegos digitales, se halló en un principio algunas dificultades para poder ingresar a la página, pero a medida que la experiencia avanzó los estudiantes accedían con mayor independencia y sin esperar las indicaciones o ayuda de la docente. Para ayudar al acceso de algunos recursos como videos o animaciones, estos se proyectaron con ayuda de un videobeam, lo que facilitó que los niños y niñas asociaran con mayor facilidad las instrucciones a imágenes que se proyectaban.

Asimismo, al llegar al espacio de reflexión, en el cierre se evidenció como los estudiantes fueron capaces de trasladar lo vivido a través de los juegos digitales (animaciones y cuentos digitales), a preguntas de reflexión y análisis que se les formularon.

La profesora les agradece la participación y para terminar dice que por favor hagan una reflexión de todas las actividades y juegos realizados en la sesión, la estudiante 3 dice "hubieron muchos obstáculos, obstáculos difíciles", la estudiante 7 dice "que no peleen porque eso hace daño y pueden hacerle chichón y pueden romperle la cabeza a un compañero, que no sean bruscos", sigue estudiante 5 "no pelear con los compañeros y pedir disculpas como aprendemos de los juegos", el estudiante 6 "no pelear con los compañeros y vivir felices para siempre" (extracto observación 5).

Así como la orientación del profesor fue constante y es importante referirse a su rol indicando que brindó pautas y realimentación constante a los estudiantes, reafirmando lo mencionado por Egenfeldt-Nielsen (como se cita en Sánchez, 2014, p. 10) quien afirma que "la presencia del profesor ha resultado fundamental para el proceso de enseñanza-aprendizaje, al poder orientar la actividad de juego hacia la consecución de los objetivos didácticos perseguidos”. 


\subsection{Con relación a las categorías emergentes}

En lo concerniente a las categorías emergentes se encontraron dos que llamaron especial atención: Internet como agente distractor y trabajo en equipo.

Con relación al Internet como agente distractor, se encontró que, en promedio, entre diez a quince estudiantes utilizaban Internet como un medio de distracción, accediendo a otras páginas web de videos, Facebook, juegos, perdiendo de vista el objetivo principal de la actividad propuesta, lo que llevó a constantes llamados de atención para orientar de nuevo a los estudiantes. Generando de esta forma la presencia de nuevas conductas disruptivas dentro del aula de clase, llevando a que la docente ejecutara acciones en pro de contrarrestar estas conductas, pero sin una estrategia clara para abordar estos casos. Tal como se registró en una de las observaciones:

Se les indica a los estudiantes ubicarse en mesa redonda, o en círculo. Algunos ya habían ingresado a otras páginas a ver videos, como el estudiante 8 y el estudiante 4, se les recuerda que no están viendo videos y se les solicita cerrar y ubicarse en la actividad (extracto registro de observación sesión 4).

$\mathrm{Al}$ revisar dentro de la literatura formal, fueron escasas las investigaciones relacionadas directamente con que el uso del Internet puede llegar a generar nuevas conductas disruptivas o distracciones en el aula de clase. Lo que abre un campo de investigación pendiente por profundizar, aunque cabe anotar que si se han realizado varias investigaciones sobre el uso de dispositivos móviles (Burns \& Lohenry, 2010; Skiba, 2011; Shrivastava \& Shrivastava, 2014; Pascuas-Rengifo, et al., 2020; Criollo-C, et al., 2021).

En lo referido al trabajo en equipo, inicialmente la experiencia se había formulado para que cada uno de los estudiantes contara con su computador, pero la infraestructura tecnológica de la institución no contaba con el correcto funcionamiento de todos los equipos, por lo que se conformaron grupos para trabajar en los juegos digitales, lo que permitió que durante la realización de las actividades se conformaran tríos o parejas y manifestaran interés por trabajar con otros compañeros, brindando así espacios para compartir logros, interés, retos y objetos. Aunque en ocasiones a algunos estudiantes les constaba compartir con sus compañeros o trabajar con la otra persona.

Lo anterior, reafirma la importancia de ser conscientes que integrar tecnologías en, lo que autores como Bosco (2013) han llamado la "hora de..., implica un apoyo institucional constante (Alonso, et al., 2014) y no se pueden catalogar como una solución definitiva o innovadora, idea que ha sido presentada como un mito en investigaciones previas (Sancho, et al., 2015). 


\section{DISCUSIÓN}

Esta investigación nos permitió determinar que los juegos digitales pueden ser un gran aporte a la generación de procesos reflexivos y analíticos, que enfocados en el fortalecimiento de competencias ciudadanas pueden llegar a generar transformaciones en el actuar e interactuar diario de los estudiantes escolares, lo que es consistente con investigaciones previas y actuales (Martínez, 2014; Castro, et. al., 2014; DeVoogd et al. 2016; Vargas y Veloza, 2017; Maldonado, 2018; Paba-Medina, et al., 2020). Lo que convierte a este tipo de escenarios digitales en estrategias útiles, desde una mirada de prevención para futuras situaciones de violencia o agresividad dentro de las aulas de clase.

Con respecto a las distintas categorías de análisis, a continuación, se realizan algunas inferencias. Sobre las normas de convivencia, se sugiere incentivar en estas edades la participación en la construcción de las normas como un ejercicio co-coinstruido que integra a todos los miembros de la comunidad educativa (Ávalos Díaz \& Berger Silva, 2021), aunque tomando en cuenta la importancia del rol docente al orientar y dirigir grupalmente, lo que es considerado como una estrategia adecuada que contribuye a la disciplina (Alonso y Martos, 2007).

Comprender y aceptar las ideas de otros en el marco del buen trato, se considera un proceso social de aprendizaje que se consolida con la interacción en diferentes contextos familiares, sociales y personales (Montes y Montoya, 2003, como se citó en Muñoz y Mondaca, 2007), lo que corrobora la importancia de brindar a los estudiantes una formación constante sobre lo que implica el buen trato hacia los demás, para lo cual los juegos digitales puede ser un primer paso que aporte a estos procesos de formación, como lo han mencionado algunas investigaciones actuales (Gallardo, et al., 2018; Puente, 2017).

Asimismo, con respecto a la empatía, es fundamental centrase en los lazos familiares que en un inicio se forman y que son la base que consolidan en el tiempo las dinámicas sociales que giran alrededor de acciones empáticas (Preston y De Waal, 2002). Por lo que se puede afirmar que los juegos digitales sólo aportan a fortalecer la empatía en los estudiantes, más no se podrían considerar como la base para formar y sustentar este tipo de habilidades personales.

\section{CONCLUSIONES}

Esta estrategia pedagógica, apoyada en juegos digitales, brinda la oportunidad de generar nuevos aprendizajes y enseñanzas en el marco de las competencias ciudadanas, pero al mis- 
mo tiempo se convierte en una nueva mirada que los profesores pueden implementar en los escenarios de clase presenciales y virtuales. Más ahora que las dinámicas educativas se han transformado en el marco de la pandemia mundial.

Es difícil establecer que sólo los juegos digitales contribuyeran a la formación en las competencias ciudadanas, debido a que los resultados muestran la influencia de otros factores y contextos cercanos, reafirmando lo que menciona la Asociación Española Contra el Cáncer AECC (2010) "dichas habilidades se pueden aprender aprovechando cada momento cotidiano en el hogar o en el aula” (p. 8).

De la misma manera, no hay que desconocer que en la formación de competencias ciudadanas "la mayor parte de las conductas se aprenden, sobre todo de los padres, de los profesores y de las personas que rodean al niño" (Asociación Española Contra el Cáncer, 2010, p. 7), por lo que se plantea para futuras investigaciones contemplar procesos de formación para padres de familia, donde las Tecnologías de la Información y la Comunicación pueden ser un medio adecuado que entrelace la escuela, la familia y el entorno externo.

Pretender que en el campo educativo se reconozcan los aportes de los juegos digitales a la formación de competencias ciudadanas, implica que el docente requiere de formación sobre estas dos áreas (Vargas Sánchez, 2019). Lo que se convierte en un campo de acción bastante amplio en el que se invita a colegios, universidades, agencias gubernamentales, a trabajar en estos aspectos y de la mano de los profesores.

Por otra parte, la literatura formal muestra escasas investigaciones relacionadas directamente con que el uso de Internet como fuente de nuevas conductas disruptivas o distracciones en el aula de clase. Lo que abre un campo de investigación pendiente por profundizar.

Finalmente, para futuras investigaciones en el área de la informática educativa, se hace necesario comprender mejor ¿Cómo los juegos digitales pueden fortalecer las competencias ciudadanas? ¿Cómo al ser fortalecidas se puede contribuir a la formación de ciudadanos digitales? y ¿En qué medida los juegos digitales pueden aportar a la formación de mejores ciudadanos?

\section{REFERENCIAS BIBLIOGRÁFICAS}

Alonso, C., Bosco, A., Corti, F. \& Rivera, P. (2014). Prácticas de enseñanza mediadas por entornos 1x1: un estudio de casos en la educación obligatoria en Cataluña. Profesorado. Revista de Currículum y Formación de Profesorado, 18(3).

18• International Journal of New Education | Núm. 8 
Alonso, C. \& Martos, S. (2007). Apartado2-1-3.asp. Open Course Ware. Universidad de Sevilla. http:// ocwus.us.es/didactica-y-organizacion-escolar/procesos-de-ensenanza-aprendizaje/asigpea/ apartados/apartado2-1-3.asp.html/

Asociación Española Contra el Cáncer AECC. (2010). Las emociones: comprenderlas para vivir mejor. http://sauce.pntic.mec.es/falcon/emociones.pdf

Ávalos Díaz, A., \& Berger Silva, C. (2021). Normas de convivencia escolar: Descripción y análisis de un proceso participativo. Estudios pedagógicos (Valdivia), 47(1), 409-429.

Bosco, A. (2013). Las TIC y la educación escolar: tiempo y espacio como obstáculos o aliados de la innovación. Investigación en la escuela, (79), 43-53.

Burns, S., \& Lohenry, K. (2010). Cellular phone use in class: Implications for teaching and learning a pilot study. College Student fournal, 44(3).

Castro, A., Borjas, M. P., Barreto, C. T. R., Herrera, M., \& Vergara, E. (2014). Recursos educativos digitales para la educación infantil (REDEI). Zona próxima: revista del Instituto de Estudios Superiores en Educación, (20), 1-21

Cha, J., Baek, Y., \& Xu, Y. (2008). Exploring Learner's variables affecting gaming achievement in digital game-based learning. In 2008 Second IEEE International Conference on Digital Game and Intelligent Toy Enhanced Learning (pp. 75-82). https://doi.org/10.1109/DIGITEL.2008.15

Criollo-C, S., Guerrero-Arias, A., Jaramillo-Alcázar, Á., \& Luján-Mora, S. (2021). Mobile Learning Technologies for Education: Benefits and Pending Issues. Applied Sciences, 11(9), 4111.

David, P., Kim, J., Brickman, J., Ran, W., \& Curtis, C. (2015). Mobile phone distraction while studying. New media \& society, 17(10), 1661-1679.

Departamento Administrativo Nacional de Estadística (DANE). (2020). Estratificación socioeconómica para servicios públicos domiciliarios. Bogotá, Colombia. https://www.dane.gov.co/index.php/servicios-al-ciudadano/servicios-informacion/estratificacion-socioeconomica\#preguntas-frecuentes

DeVoogd, K., Lane-Garon, P., \& Kralowec, C. A. (2016). Direct Instruction and Guided Practice Matter in Conflict Resolution and Social-Emotional Learning. Conflict Resolution Quarterly, 33 (3), 279-296.

Gallardo, I., Aguasanta, M. E., Benjamin, A. C. \& Saiz, H. (2018). Análisis de recursos digitales en Educación Infantil (Capitulo 12. Págs. 140-149). En Jiménez Hernández, A. y Iglesias Villarán, M. (Coords) (2018). Cultura de Paz y buen trato a la Infancia. Huelva: Consejo Independiente de Protección de la Infancia (CIPI). https://roderic.uv.es/handle/10550/68009

Gómez, M. (2006). Introducción a la Metodología de la investigación Científica. Córdoba, Argentina: Editorial Brujas. 1 ed. 
Gros, B. (coord.). (2008). Videojuegos y aprendizaje. Barcelona, España: Grao.

Hernández, R., Fernández, C., \& Baptista, P. (2010). Metodología de la Investigación. México: McGrawHill. 5ta ed.

Jares, X. (2001). Educación y conflicto. Guía de educación para la convivencia. Madrid, España: Editorial Popular S.A.

Maldonado, M. E. (2018). El aula, espacio propicio para el fortalecimiento de competencias ciudadanas y tecnológicas. Sophia, 14(1), 39-50.

Martínez, S. (2014). Estudio de caso: Fortalecimiento de competencias ciudadanas a través de estrategias mediadas por las tecnologías de la información y la comunicación. [Tesis de maestría]. Universidad de La Sabana, Chía, Colombia. http://intellectum.unisabana.edu.co:8080/jspui/bitstream/10818/11587/1/Sayana\%20Malfasi \%20Martinez \%20\%28tesis \%29.pdf

Ministerio de Educación Nacional. (2004). Estándares básicos de competencias ciudadanas: Formar para la ciudadanía... ¡Sí es posible! Lo que necesitamos saber y saber hacer. Serie Guías No. 6. http:// www.mineducacion.gov.co/cvn/1665/articles-75768 archivo pdf.pdf

Ministerio de Educación Nacional. (2012). Competencias Ciudadanas. http://www.mineducacion.gov. co/1621/w3-article-235147.html

Ministerio de Educación Nacional. (2014). Guía No. 49. Guías pedagógicas para la convivencia escolar. Ley 1620 de 2013 - Decreto 1965 de 2013. Bogotá, Colombia. https://www.mineducacion.gov. co/1759/w3-article-339480.html? noredirect $=1$

Muñoz, L. G. (2018). Los derechos humanos de los niños: ciudadanía más allá de las “3Ps”. Sociedad e infancias, (2), 17-37.

Muñoz, T., \& Mondaca, B. (2007). Talleres de capacitación de convivencia y buen trato para educadores de niños con necesidades educativas especiales. Electronic fournal of Research in Educational Psychology [online], (5).

Ojeda, M. J. C., Herrera, D. G. G., Mediavilla, C. M. Á., \& Álvarez, J. C. E. (2020). El juego como motivación en el proceso de enseñanza aprendizaje del niño. Revista Arbitrada Interdisciplinaria Koinonía, 5(1), 430-448.

Paba-Medina, Acosta-Medina \& Torres-Barreto. (2020). Priorización de competencias ciudadanas en un contexto gamificado. Panorama, 14(27), 51-72.

Pascuas-Rengifo, Y. S., García-Quintero, J. A., \& Mercado-Varela, M. A. (2020). Dispositivos móviles en la educación: tendencias e impacto para la innovación. Revista Politécnica, 16(31), 97-109. 
Pizarro, G. \& Cordero, D. (2013). Las TIC: Una herramienta tecnológica para el desarrollo de las competencias lingüísticas en estudiantes universitarios de una segunda lengua. Revista Electrónica Educare. 17(3), 277-292.

Puente, R. P. (2017). Talleres de co-creación para promover la equidad y la inclusión de las personas con alzheimer a través del juego y las TIC. Comunidades sostenibles: dilemas y retos desde el trabajo social, (1337-1343).

Preston, S., \& De Waal, F. (2002). Empathy: Its ultimate and proximate bases. Behavioral and brain sciences, 25(01), 1-20.

Quiceno, F., Betancur, M., \& Rojas, H. M. (2020). La enseñanza en ciudadanía: nuevas exigencias para la escuela. Sophia, 16(1), 65-75.

Reyes, K. (2015). El buen trato en las relaciones de cuidado con niños y niñas. Chile crece contigo. http:// www.crececontigo.gob.cl/columna/el-buen-trato-en-las-relaciones-de-cuidado-con-ninos-yninas/

Rodríguez, F., \& Santiago, R. (2015). Gamificación: Cómo motivar a tu alumnado y mejorar el clima en el aula. Barcelona, España: Editorial Oceano.

Roig, R., Mengual, S., \& Quinto, P. (2015). Primary Teachers' Technological, Pedagogical and Content Knowledge. Comunicar, 23(45), 151-159. doi: 10.3916/C45-2015-16

Rosi, A., Dall'Asta, M., Brighenti, F., Del Rio, D., Volta, E., Baroni, I. \& Scazzina, F. (2016). The use of new technologies for nutritional education in primary schools: a pilot study. Public Health, 140, 50-55.

Ruiz, A. y Chaux E. (2005). La formación de competencias ciudadanas. Bogotá: Ascofade.

Sánchez, J. (2014). Motivación y aprendizaje de ciencias sociales en estudiantes de PCPI con un videojuego a través de la pizarra digital. Un estudio de caso. Revista Científica de Opinión y divulgación DIM Didáctica, Innovación y Multimedia. 10 (30), 1-15.

Sancho, J., Bosco, A., Alonso, C. \& Sánchez, J. (2015). Formación del profesorado en Tecnología Educativa: de cómo las realidades generan los mitos. Revista Latinoamericana de Tecnología Educativa-RELATEC, 14(1), 17-30.

Santiago, R., Trabaldo, S., Kamijo, M. \& Fernández, A. (2015). Mobile Learning: Nuevas Realidades en el Aula. Barcelona, España: Editorial Océano.

Shrivastava, A., \& Shrivastava, M. (2014). Classroom distraction due to mobile phones usage by students: college teachers' perceptions. International fournal of Computer and Information Technology, 3(3), 638-642. 
Skiba, D. (2011). On the HorizonMobile Devices: Are They a Distraction or Another Learning Tool?. Nursing Education Perspectives, 32(3), 195-197.

Stake, R. (1998). Investigación con estudio de casos. Madrid, España: Morata.

Torrego, J. \& Moreno, J. (2003). Convivencia y disciplina en la escuela el aprendizaje de la democracia. Madrid, España: Alianza Editorial S.A.

UNESCO. (2018). School violence and bullying: Global status and trends, drivers and consequences. Paris, Francia. http://www.infocoponline.es/pdf/BULLYING.pdf

UNESCO. (2013). Enfoques estratégicos sobre las TIC en educación en América Latina y el Caribe. Santiago, Chile. http://www.unesco.org/new/fileadmin/MULTIMEDIA/FIELD/Santiago/pdf/TICSenfoques-estrategicos-sobre-TICs-ESP.pdf

Universidad Distrital Francisco José de Caldas. (2016). En 2016 se presentaron 30.241 situaciones que afectaron el clima escolar [Entrevista en línea]. Bogotá, Colombia. http://laud.udistrital.edu.co/ noticias/en-2016-se- presentaron-30241-situaciones-que-afectaron-el-clima-escolar

Urrea Monclús, A., Coiduras Rodríguez, J. L., Alsinet, C., Balsells, M., Guadix García, I., \& Belmonte Castell, O. (2018). Derechos de la Infancia y Ciudadanía Global: una aproximación desde la comunidad educativa. Sociedad e Infancias, (2), 127-146.

Vargas, A., \& Veloza, L. (2017). Education in Conflict Resolution Using ICT: A Case Study in Colombia. Fournal of Cases on Information Technology (FCIT), 19(2), 29-43.

Vargas Sánchez, A. D. (2019). Educación para la convivencia y la cultura de paz a través de las TAC: un estudio multicasos en el contexto bogotano. [Tesis doctoral], Universitat Autònoma de Barcelona, España.

Wood, E., Specht, J., Willoughby, T., \& Mueller, J. (2008). Integrating computer technology in early childhood education environments: Issues raised by early childhood educators. Alberta fournal of Educational Research, 54(2), 210.

Wood, E., Zivcakova, P. G., Archer, K., De Pasquale, D. \& Nosko, A. (2012). Examining the impact of off-task multi-tasking with technology on real-time classroom learning. Computers \& Education, 58, pp. 365-374. 